\title{
2D:4D RATIO DIFFERS IN ISCHEMIC STROKE: A SINGLE CENTER EXPERIENCE
}

Background: The index to ring finger length (2D:4D) ratio is a proxy biomarker for prenatal exposure of sex hormones. Sex hormones are associated with the pathogenesis of ischemic stroke. The purpose of the study was to demonstrate the association between 2D:4D and ischemic stroke.

Methodology: This study retrospectively reviewed the data of 100 patients with first ever ischemic stroke between September, 2016 and June, 2017. The lengths of index finger and ring finger of both hands were measured using electronic calipers and calculated for 2D:4D ratios. Receive operating characteristic (ROC) mode was used to detect predicting performance of 2D:4D ratios for ischemic stroke.

Results: 2D:4D ratios in ischemic stroke patients were higher than controls in both hands $(\mathrm{P}<0.05)$, except right $2 \mathrm{D}: 4 \mathrm{D}$ ratio in females. The $\mathrm{ROC}$ analyses showed that the area under the curve (AUC) were 0.635 ( $95 \% \mathrm{Cl}: 0.527-$ $0.743)$ for left $2 \mathrm{D}: 4 \mathrm{D}$ ratio, and 0.647 for right $(95 \% \mathrm{Cl}: 0.539-0.755)(\mathrm{P}<0.05)$. The AUC of left and right $2 \mathrm{D}: 4 \mathrm{D}$ ratio in male were 0.667 (95\% Cl: 0.514-0.820) and 0.670 (95\% Cl: 0.519-0.822) $(\mathrm{P}<0.05)$. In female, no significance were found in ROC analysis. And there were no correlation between 2D:4D value and stroke severity $(P>0.05)$.

Conclusions: The current study indicated that the diagnostic value of $2 \mathrm{D}: 4 \mathrm{D}$ ratio was limited in ischemic stroke. Further research is required to explore the role of it in screening ischemic stroke.

Keywords

-2D:4D ratio $\cdot$ ischemic stroke $\cdot$ biomarker $\cdot$ finger length

\section{Introduction}

The ratio of the index and ring fingers (2D:4D ratio) is a proposed biomarker for prenatal sex hormone exposure. The higher ratio in women is associated with greater prenatal testosterone and lower estrogen exposure [1,2]. It is also related to a wide range of diseases. Right and left hand 2D:4D ratios are lower in primary brain tumor patients relative to healthy individuals, with greater prenatal testosterone exposure in patients [3]. And patients with coronary artery disease are likely to have higher 2D:4D ratio than controls in both hands [4]. Ischemic stroke (IS) is recognized as a sexually dimorphic disorder, with a relatively lower stroke incidence in women [5]. It usually induces disability and mortality among the encounters all over the world $[6,7]$. Epidemiological and clinical studies have indicated the altered testosterone and estrogen levels in IS $[5,8]$. The sex hormones may account for the stroke sensitivity of among the population. However, no studies are available to detect the relationship between 2D:4D ratio and IS. We here took this study to demonstrate the issue.

\section{Materials and Methods}

\section{Patient selction}

Written informed consent from all participants were obtained. This study was approved by the Ethics Committee of the institution in Wannan Medical College and was performed in accordance with the principles of Declaration of Helsinki. In this cross-sectional retrospective study, we recruited 100 subjects from the Department of Neurology, Yijishan Hospital between September 2016 and June 2017. Yijishan Hospital is a stroke center in East China Region, covering approximately 8 million people. All cases were form Han ethnic group. Individuals with former hand lesions, infertility, endocrine disorders, genetic diseases, and
Ke Yang,

XianHui Ding,

ZhiMing Zhou,

XiaoLei Shi*

Department of Neurology, The First Affiliated Hospital, Yijishan Hospital of Wannan Medical College, Wuhu, China

Ke Yang and Xianhui Ding contributed equally to this study.

Received 11 August 2018 accepted 24 September 2018

*E-mail: shixiaolei05@gmail.com

left handers were excluded. Patients with first ever IS were enrolled in patient group. An acute IS was confirmed by computed tomography or magnetic resonance imaging of the brain within 48 hours onset, on cases with neurological deficits. Subjects with IS and other central nervous system diseases history, that might influence the reliability of this study, were excluded. Two experienced physicians were responsible for inclusion and exclusion, independently. Any disagreement was determined by the senior neurologist (the corresponding author). The agreement between the two physicians were $96 \%$ for the inclusion and $95 \%$ for the exclusion.

\section{Measurement of Finger Lengths and} National Health Institute Stroke Scale (NIHSS)

Two independent examiners measured finger lengths, without acknowledgement of the medical diagnosis of the volunteers. The lengths 
of index and ring fingers were measured on the palmar surface of hands from the basal crease proximal to the palm to the tip of the finger, using electronic calipers as previously reported [9]. Admission NIHSS was determined by two experienced stroke neurologists, who were not relevant to this study. The NIHSS includes the following domains: level of consciousness, eye movements, integrity of visual fields, facial movements, arm and leg muscle strength, sensation, coordination, language, speech and neglect. Each domain is scored on an ordinal scale ranging from $0-2,0-3$, or $0-4$, with a total score from 0-42. Data were calculated, retested and recorded on computer. All these performers were blind to the design of the project and the further statistical analysis.

\section{Statistical Analysis}

Statistical analyses were performed using the SPSS statistical software (SPSS 19.0, USA). The results were expressed as mean \pm SD. Differences between the variables were determined by two-tailed Student's $t$ test. Receive operating characteristic (ROC) mode was used to detect predicting performance of 2D:4D ratios for IS. Spearman correlation analysis was used to study the relationship between 2D:4D ratios and NIHSS. $P<0.05$ was regarded as statistically significant.

\section{Results}

The baseline characteristics were shown in Table 1.100 cases were included in this study, with 50 controls $(25$ males and 25 females, average age: $60.36 \pm 12.29$ years old) and $50 \mathrm{IS}$

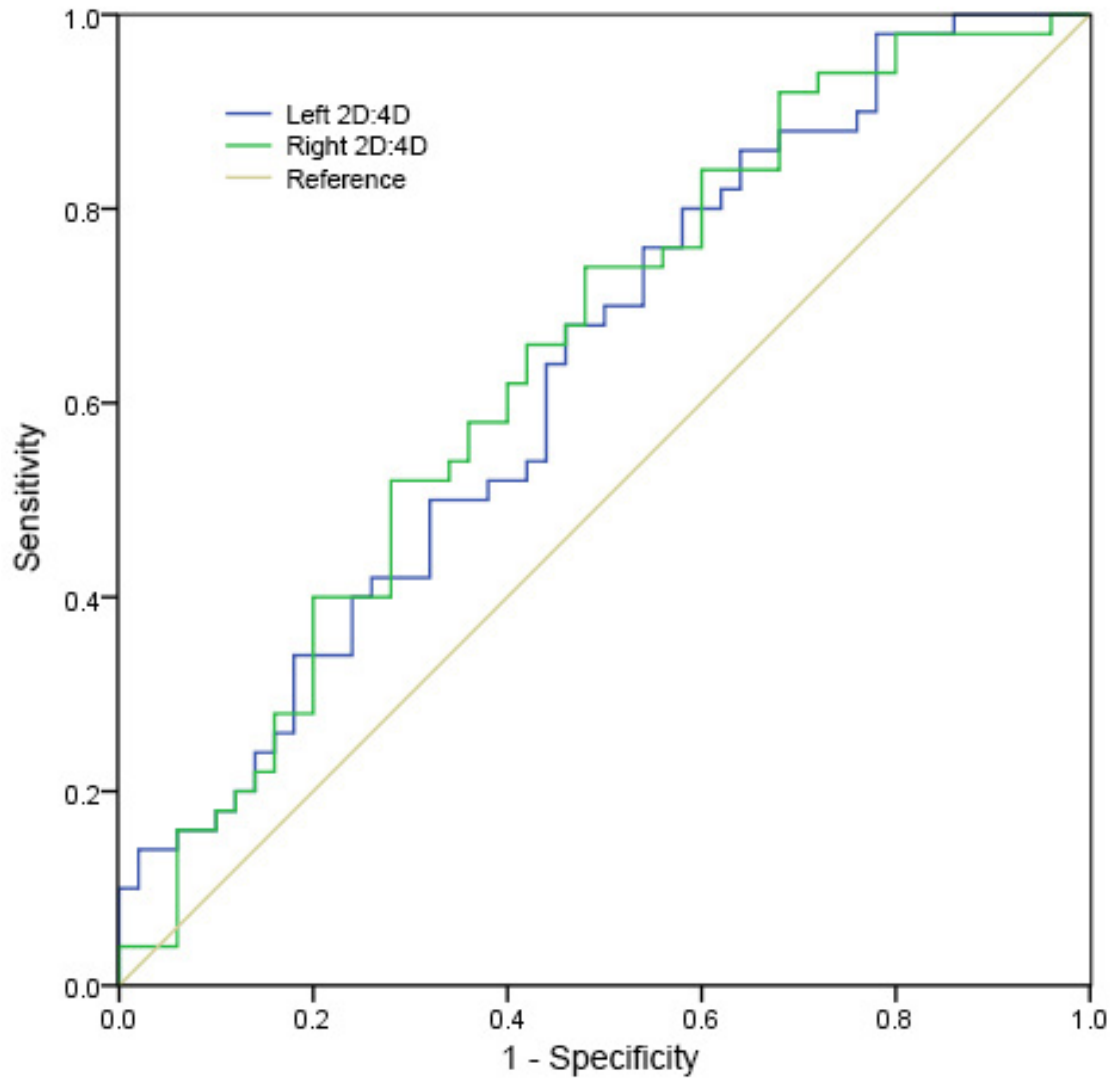

Figure 1: The area under the curve (AUC) of left 2D:4D in all.

participants ( 25 males and 25 females, average age: $64.38 \pm 13.36$ years old). There were no significant differences of age, atrial fibrillation and alcohol history between controls and patients $(P>0.05)$. A significance was found in hypertension, diabetes, hyperlipemia, smoking between controls and sufferers $(P<0.05)$
As shown in Table 2, 2D:4D ratios of both hand were markedly higher than that in controls (Pleft $=0.002 ; P_{\text {right }}=0.006$ ). The same trend was found in male ones (Pleft $=0.023$; $\left.P_{\text {right }}=0.043\right)$. For females, left $2 \mathrm{D}: 4 \mathrm{D}$ ratios of patients were significantly higher over healthy ones $(P=0.036)$, but not in right hand $(P=0.057)$.

Table 1. The baseline characteristics of this study. Con, Control; IS, Ischemic Stroke; y, year; n, number. Con, Control; IS, Ischemic Stroke; $y$, year; $n$, number.

\begin{tabular}{|c|c|c|c|c|c|c|}
\hline & \multicolumn{2}{|c|}{ All $(n=100)$} & \multicolumn{2}{|c|}{ Male $(n=50)$} & \multicolumn{2}{|c|}{ Female $(n=50)$} \\
\hline & Con & IS & Con & IS & Con & IS \\
\hline Age (y) & $60.36 \pm 12.29$ & $64.38 \pm 13.36$ & $59.00 \pm 12.34$ & $61.76 \pm 13.40$ & $62.68 \pm 12.19$ & $67.00 \pm 13.06$ \\
\hline Hypertension (n) & 18 & 32 & 8 & 17 & 10 & 15 \\
\hline Diabetes (n) & 10 & 30 & 7 & 17 & 3 & 13 \\
\hline Atrial Fibrillation & 10 & 11 & 4 & 3 & 6 & 8 \\
\hline Hyperlipemia & 15 & 38 & 8 & 25 & 7 & 13 \\
\hline Smoking (n) & 7 & 16 & 7 & 15 & 0 & 1 \\
\hline Alcohol (n) & 5 & 11 & 5 & 11 & 0 & 0 \\
\hline NIHSS & - & $4.90 \pm 5.50$ & - & $5.40 \pm 7.12$ & - & $4.40 \pm 3.24$ \\
\hline
\end{tabular}

Con, Control; Is, Ischemic Stroke; y, year; n, number. 
Table 2. The association between 2D:4D ratio and IS. Con, Control; IS, Ischemic Stroke.

\begin{tabular}{ccccccc}
\hline & & All & \multicolumn{3}{c}{ Male } & \multicolumn{2}{c}{ Female } \\
\hline & left 2D:4D & right 2D:4D & left 2D:4D & right 2D:4D & left 2D:4D & right 2D:4D \\
\hline Con & $0.9475 \pm 0.0340$ & $0.9444 \pm 0.0287$ & $0.9391 \pm 0.0379$ & $0.9387 \pm 0.0301$ & $0.9559 \pm 0.0279$ & $0.9502 \pm 0.0265$ \\
IS & $0.9686 \pm 0.0331$ & $0.9598 \pm 0.0255$ & $0.9629 \pm 0.0337$ & $0.9550 \pm 0.0251$ & $0.9743 \pm 0.0321$ & $0.9646 \pm 0.0256$ \\
$P$ & 0.002 & 0.006 & 0.023 & 0.043 & 0.036 & 0.057 \\
\hline
\end{tabular}

The ROCs analyzing showed that the area under the curve (AUC) of left 2D:4D in all was 0.635 (95\%Cl: $0.527-0.743, P=0.020)$, and it was 0.647 for right hand $(95 \% \mathrm{Cl}: 0.539-0.755, P=$ 0.011 ) (Figure 1). The optimal cutoff point of left 2D:4D ratio for AIS was 0.9570 with sensitivity of $68.0 \%$ and specificity of $54.0 \%$. For right hand, the point was 0.9445 with sensitivity of $74.0 \%$ and specificity of $52.0 \%$. We discovered that AUCs of $2 \mathrm{D}: 4 \mathrm{D}$ ratio in male were 0.667 (95\%Cl: $0.514-0.820, P=0.043$ ) (left) and 0.670 (95\%Cl: 0.519-0.822, $P=0.039$ ) (right) (Figure 2). The optimal cutoff points of $2 \mathrm{D}: 4 \mathrm{D}$ value for male were 0.9570 with $60.0 \%$ sensitivity and $72.0 \%$ specificity (left), and 0.9445 with sensitivity of $76.0 \%$ and specificity of $60.0 \%$ (right). However, AUCs of both hands in female were not statistically significant (Pleft $=0.109$, Pright $=0.138$ ).

We also assessed the relationship between 2D:4D ratios and NIHSS. No differences were found between 2D:4D ratios and NIHSS (All: 4.90 \pm 5.50 , Pleft $=0.803$, Pright $=0.565$; Male: $5.40 \pm 7.12$, Pleft $=0.960$, Pright $=0.710$; Female: $4.40 \pm 3.24$, Pleft $=0.984$, Pright $=0.592$ ).

\section{Discussion}

The current study was the first to study the association between 2D:4D ratio and IS. We found that stroke patients had a higher 2D:4D ratio in both hands. The same trends were found in both hands of male, and in female left hand.

$2 \mathrm{D}: 4 \mathrm{D}$ ratio is affected by prenatal sex hormones exposure and it acts as the symbol of sexual orientation of adults [9]. The ratio tends to be higher in female, being associated with greater prenatal testosterone and lower estrogen exposure. Epidemiological and clinical studies have indicated men intend to have a higher incidence of IS $[11,12]$. The most common biological explanation for

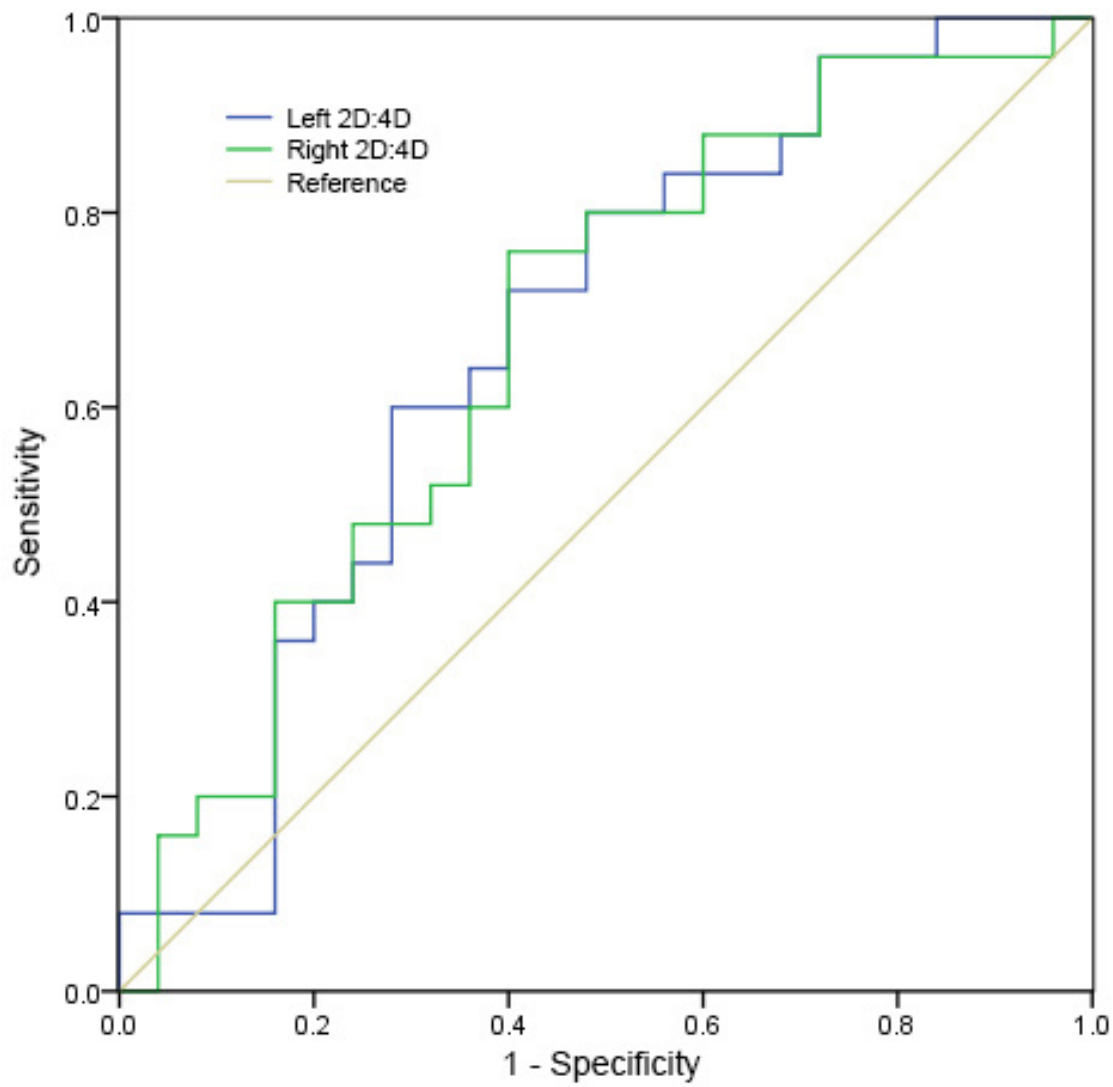

Figure 2: The area under the curve (AUC) of left 2D:4D in male.

this phenomenon may be attributed to sex hormones. Atherosclerosis of intracranial artery and thrombus formation is the core features of IS. Testosterone increases platelet aggregation and subsequent arterial thrombus formation [13]. Estrogen has very potent effects on endothelial cells through promoting dilation and blood flow [14]. An extremely lower endogenous testosterone was associated with higher risk of IS [15]. And postmenopausal women receiving estrogen therapy had a lower IS rate than those without intervention [16]. Also, sex hormones lead to microglia and astrocyte activation $[17,18]$, which are major contributing cells in neuroinflammation in response to various insults [19-21]. The inflammatory status would exacerbate the atherosclerosis in vascular arteries [22]. Some researchers have confirmed the link between 2D:4D ratio and arterial atherosclerosis through biopsy [23].

We believed that there might be connections between 2D:4D and IS. The present study indicated that men tended to have a higher $2 \mathrm{D}: 4 \mathrm{D}$ ratio in both hands. This is consistent with the previous findings in coronary heart disease. In that study, men with coronary heart disease shared a 
higher ratio of 2D:4D $[24,25]$. This might be attributed to the cause of atherosclerosis changes in both disorders. However, the same trends only existed in left, but not right hand among women, indicating potential sex differences in this disease. More studies should be done to discover this matter. Also, we used electronic calipers to directly measure the lengths of fingers, instead of calculating them from photocopies and scans. This ensured the reliability and accuracy, as previously proved in a recent review [26].

NIHSS is a common tool for the assessment of stroke severity. It was originally developed in 1989 [27] and now widely applied in various studies [2830]. It was used to assess the relationship between
2D:4D ratios and stroke severity. It indicated that 2D:4D was not associated with the severity. It worked as a qualitative, not a quantitative tool for brain ischemia. High 2D:4D ratio is a possible biomarker for IS. Further research is required to validate this association in different populations in screening of patients' susceptibility to develop IS.

A significant association was not found between 2D:4D ratio and IS in the current study. $\mathrm{ROC}$ analysis indicated its role in the diagnostic role of 2D:4D ratio in IS, but with limited sensitivity and specificity. It only indicated the possible place of it in the early understanding of the disorder.

The current study indicated that stroke patients had a higher 2D:4D ratio in both hands.
The diagnostic value of 2D:4D ratio was limited in IS. Further research is required to explore the role of it in screening IS.

\section{Conflict of interest}

None of the authors has a conflict of interest to declare.

\section{Funding}

This study was supported by grants from Talent Scientific Research Start-up Foundation of Yijishan Hospital, Wannan Medical College (Grant No. YR201603), Youth and Middle Foundation of Wannan Medical College (Grant No. KY86700312) and National Natural Science Foundation of China (Grant No. 81701061).

References

[1] Zheng Z, Cohn MJ. Developmental basis of sexually dimorphic digit ratios. Proc Natl Acad Sci U S A 2011 2011-09-27;108(39):16289-94.

[2] Manning JT. Resolving the role of prenatal sex steroids in the development of digit ratio. Proc Natl Acad Sci U S A 2011 2011-0927;108(39):16143-4.

[3] Bunevicius A, Tamasauskas S, Deltuva VP, Tamasauskas A, Sliauzys A, Bunevicius R. Digit ratio (2D:4D) in primary brain tumor patients: A case-control study. Early Hum Dev 2016 2016-12-01;103:205-8.

[4] Lu H, Ma Z, Zhao J, Huo Z. Second to fourth digit ratio (2D:4D) and coronary heart disease. Early Hum Dev 2015 2015-07-01;91(7):41720.

[5] Suzuki S, Brown CM, Wise PM. Neuroprotective effects of estrogens following ischemic stroke. Front Neuroendocrinol 2009 2009-0701;30(2):201-11.

[6] Christophe BR, Mehta SH, Garton AL, Sisti J, Connolly EJ. Current and future perspectives on the treatment of cerebral ischemia. Expert Opin Pharmacother 2017 2017-04-01;18(6):573-80.

[7] Cruz-Flores S. Acute Stroke and Transient Ischemic Attack in the Outpatient Clinic. Med Clin North Am 2017 2017-05-01;101(3):47994.

[8] Jeppesen LL, Jorgensen HS, Nakayama H, Raaschou HO, Olsen TS, Winther K. Decreased serum testosterone in men with acute ischemic stroke. Arterioscler Thromb Vasc Biol 1996 1996-06-01;16(6):749-54.

[9] Fink B, Manning JT, Neave N. The 2nd-4th digit ratio (2D:4D) and neck circumference: implications for risk factors in coronary heart disease. Int J Obes (Lond) 2006 2006-04-01;30(4):711-4.

[10] Ventura T, Gomes MC, Pita A, Neto MT, Taylor A. Digit ratio (2D:4D) in newborns: influences of prenatal testosterone and maternal environment. Early Hum Dev 2013 2013-02-01;89(2):107-12.

[11] Barker-Collo S, Bennett DA, Krishnamurthi RV, Parmar P, Feigin VL,
Naghavi $M$, et al. Sex Differences in Stroke Incidence, Prevalence, Mortality and Disability-Adjusted Life Years: Results from the Global Burden of Disease Study 2013. Neuroepidemiology 2015 2015-0120;45(3):203-14

[12] Yatsuya H, Folsom AR, Yamagishi K, North KE, Brancati FL, Stevens J. Race- and sex-specific associations of obesity measures with ischemic stroke incidence in the Atherosclerosis Risk in Communities (ARIC) study. Stroke 2010 2010-03-01;41(3):417-25.

[13] Glueck CJ, Wang P. Testosterone therapy, thrombosis, thrombophilia, cardiovascular events. Metabolism 2014 2014-08-01;63(8):989-94.

[14] Tarhouni K, Guihot AL, Vessieres E, Procaccio V, Grimaud L, Abraham $\mathrm{P}$, et al. Estrogens are needed for the improvement in endotheliummediated dilation induced by a chronic increase in blood flow in rat mesenteric arteries. Vascul Pharmacol 2016 2016-05-01;80:35-42.

[15] Holmegard HN, Nordestgaard BG, Jensen GB, Tybjaerg-Hansen A, Benn M. Sex Hormones and Ischemic Stroke: A Prospective Cohort Study and Meta-Analyses. J Clin Endocrinol Metab 2016 2016-0101;101(1):69-78.

[16] Wassertheil-Smoller S, Hendrix SL, Limacher M, Heiss G, Kooperberg $C$, Baird A, et al. Effect of estrogen plus progestin on stroke in postmenopausal women: the Women's Health Initiative: a randomized trial. JAMA 2003 2003-05-28;289(20):2673-84.

[17] Drew PD, Chavis JA. Female sex steroids: effects upon microglial cell activation. J Neuroimmunol 2000 2000-11-01;111(1-2):77-85.

[18] Fernandez-Galaz MC, MorschI E, Chowen JA, Torres-Aleman I, Naftolin F, Garcia-Segura LM. Role of astroglia and insulin-like growth factor-I in gonadal hormone-dependent synaptic plasticity. Brain Res Bull 1997 1997-01-19;44(4):525-31.

[19] Xu X, Cai X, Zhu Y, He W, Wu Q, Shi X, et al. MFG-E8 inhibits Abetainduced microglial production of cathelicidin-related antimicrobial 
peptide: A suitable target against Alzheimer's disease. Cell Immunol 2018 2018-05-24.

[20] Shi X, Cai X, Di W, Li J, Xu X, Zhang A, et al. MFG-E8 Selectively Inhibited Abeta-Induced Microglial M1 Polarization via NF-kappaB and PI3K-Akt Pathways. Mol Neurobiol 2017 2017-12-01;54(10):7777-88.

[21] Shao YY, Li B, Huang YM, Luo Q, Xie YM, Chen YH. Thymoquinone Attenuates Brain Injury via an Anti-oxidative Pathway in a Status Epilepticus Rat Model. Transl Neurosci 2017 2017-01-01;8:9-14.

[22] Xing J, Lu J. Effects of mTOR on Neurological Deficits after Transient Global Ischemia. TransI Neurosci 2017 2017-01-20;8:21-6.

[23] Ozdogmus O, Cakmak YO, Coskun M, Verimli U, Cavdar S, Uzun I. The high 2D:4D finger length ratio effects on atherosclerotic plaque development. Atherosclerosis 2010 2010-03-01;209(1):195-6.

[24] Kyriakidis I, Papaioannidou P, Pantelidou V, Kalles V, Gemitzis K. Digit ratios and relation to myocardial infarction in Greek men and women. Gend Med 2010 2010-12-01;7(6):628-36.

[25] Wu XL, Yang DY, Chai WH, Jin ML, Zhou XC, Peng L, et al. The ratio of second to fourth digit length (2D:4D) and coronary artery disease in a
Han Chinese population. Int J Med Sci 2013 2013-01-20;10(11):15848.

[26] Ribeiro E, Neave N, Morais RN, Manning JT. Direct versus indirect measurement of digit ratio (2D: 4D) a critical review of the literature and new data. Evolutionary Psychology 2016 2016-0101;14(1):2125747160.

[27] Nilanont Y, Komoltri C, Saposnik G, Cote R, Di Legge S, Jin Y, et al. The Canadian Neurological Scale and the NIHSS: development and validation of a simple conversion model. Cerebrovasc Dis 2010 201001-20;30(2):120-6.

[28] Martin-Schild S, Albright KC, Tanksley J, Pandav V, Jones EB, Grotta JC, et al. Zero on the NIHSS does not equal the absence of stroke. Ann Emerg Med 2011 2011-01-01;57(1):42-5.

[29] Braksick SA, Wijdicks EF. An NIHSS of 0 and a Very Disabling Stroke. Neurocrit Care 2016 2016-10-26.

[30] Guo C, Shi X, Ding X, Zhou Z. Analysis of Radiation Effects in Digital Subtraction Angiography of Intracranial Artery Stenosis. World Neurosurg 2018 2018-04-21. 